\title{
PERCEPTION BASED BINARY IMAGE WATERMARKING
}

\author{
Anthony T.S. Ho, Niladri B. Puhan, Pina Marziliano, Anamitra Makur, Y. L. Guan \\ School of Electrical and Electronic Engineering \\ Nanyang Technological University, Singapore \\ E-mail: ETSHO@ntu.edu.sg
}

\begin{abstract}
The use of a suitable perceptual model is necessary to minimize the visual distortion in the marked images, because minor modification to the pixels can be perceptible since the pixels are either black or white. In this paper, a new perceptual model is proposed for binary images that is useful for data hiding applications. In our model, the distortion that occurs after flipping a pixel is estimated on the novel curvature-weighted distance difference $(C W D D)$ measure between two contour segments. Through subjective tests the perceptual measure is validated and highly correlated with human perception.
\end{abstract}

\section{INTRODUCTION}

Ease of perfect copying and editing in the digital domain raises the question of the security and legal usage of multimedia data. So the protection of ownership and the prevention of unauthorized tampering of images and video have become important topics. For images in which the pixels take on only a limited number of values, hiding data without causing visible artifacts becomes more difficult. So the watermarking algorithm should use valid perceptual model for achieving high watermark capacity. Low et al $[1,2]$ proposed robust data hiding methods in formatted document images based on imperceptible line and word shifting. Their methods were applied to embed information in text images for bulk electronic publications. The line shifting method has low data hiding capacity as compared to the word shifting method but the embedded data is more robust to photocopying, scanning and printing process. Koch and Zhao [3] proposed a data hiding algorithm in which a data bit ' 1 ' is embedded if the percentage of white pixels was greater than a given threshold, and a data bit ' 0 ' is embedded if the percentage of white pixels was less than another given threshold. In [4], the proposed algorithm slightly modified interword spaces so that different lines across a text act as sampling points of a sine wave. After the modification, the average spaces of various lines have the characteristics of a sine wave and the wave constituted a mark. This algorithm claimed to withstand different noise and distortion attacks. Wu et al [5] hid data in a binary image using a hierarchical model in which human perception was taken into consideration. In a block, modifying the total number of black pixels to be either odd or even embeds data bits. Lu et al proposed an objective distortion measure known as the distance-reciprocal distortion measure (DRDM) for binary document images [6] that could be used for performance comparison in data hiding applications. Traditional objective distortion measures like mean square error (MSE), signal-to-noise ratio (SNR), and peak signalto-noise ratio (PSNR) are not well correlated with human perception for binary images. All three measures only take the number of flipped pixels into account and the distortions in the binary images can be different even if the number of flipped pixels is the same.

The paper is organized as follows: Section 2 presents a contour- based metric for the proposed perceptual model. The subjective experiments described in Section 3 are used to validate the perceptual model and these results will be presented in Section 4. Finally some conclusions are given in Section 5.

\section{PROPOSED PERCEPTUAL MODEL}

We propose a new perceptual model based on the curvature-weighted distance difference $(C W D D)$ measure between two contour segments.

Definition 1: Contour segment

A contour segment with a set of $n$ pixels $\left\{p_{i}\right\}, i=0,1, \ldots, n-1$ can be represented by $(n-1)$ 
chain codes $\left\{c_{i}\right\}, i=1,2, \ldots, n-1$, where $c_{i}$ is the direction from pixel $p_{i-1}$ to $p_{i}$.

A pixel is considered as a contour pixel, if it has at least one 4-connected neighbor pixel of opposite value. The idea behind the model is simple: when a black contour pixel is flipped, the distortion that we are able to perceive is an intrusion of the character boundary and if a white contour pixel is flipped we perceive the opposite effect, i.e. protrusion of the character boundary. The amount of intrusion or protrusion can be measured by the change in the contour segment that passes through the flipped pixel. If the length of the contour segment increases or decreases after flipping, the Euclidean distance along the contour segment would reflect this change. High change in curvature relates to more visibility of distortion. So both the Euclidean distance and the curvature are used in measuring the level of distortion in the binary images in this model

Definition 2: Curvature

Given an ordered set of pixels $P=\left\{p_{i}\right\}, i=0, \ldots, n-1$, the curvature at pixel $p_{i} \in P$ is given by

$\alpha_{p_{i}}= \begin{cases}\left(180^{0}-\theta_{i}\right) & i=1,2, \ldots, n-2 \\ 0^{0} & i=0, n-1\end{cases}$

where $\theta_{i} \in\left[0,180^{\circ}\right]$, is the angle between the continuous segments $\left[p_{i-1}, p_{i}\right]$ and $\left[p_{i}, p_{i+1}\right]$.

Since the contour segment is represented by the chain code, this code can be efficiently applied to derive $d_{i}$, the Euclidean distance between two pixels $p_{i-1}$ and $p_{i}$. The curvature value $\alpha_{p_{i}}$ at pixel $p_{i}$ is 0 for $i=0$ and $i=n-1$. For $i=1, \ldots, n-2$ it is computed from the chain codes $c_{i}$ and $c_{i+1}$ as:

$\alpha_{p_{i}}= \begin{cases}\beta & \beta \leq 180^{\circ} \\ \left(360^{\circ}-\beta\right) & \text { otherwise }\end{cases}$

where

$\beta=\left|c_{i+1}-c_{i}\right| \times 45^{0}$

Equation (3) is derived based on the directional property of the chain code that corresponds to a specific angle in multiples of $45 \mathrm{deg}$. The chain code is then applied to the contour from the start pixel until the end pixel. After obtaining the curvature value at each pixel, a weight sequence $\left\{w_{i}\right\}, i=0,1, \ldots, n-1 \quad$ is experimentally defined by the following:

$$
w_{i}= \begin{cases}1 & \alpha_{p_{i}}=0^{0} \\ 1.5 & \alpha_{p_{i}}=45^{0} \\ 3 & \alpha_{p_{i}}=90^{\circ} \\ 4.5 & \alpha_{p_{i}}=135^{\circ} \\ 5.32 & \alpha_{p_{i}}=180^{\circ}\end{cases}
$$

In Eq. $4, w_{i}$ is chosen to be monotonic to $\alpha_{p_{i}}$ and the last weight is chosen to be 5.32 such that the maximum possible value of $C W D D$ measure between two five-pixel long contour segments is 20 . The curvature-weighted distance $\left(D_{\alpha}\right)$ of a contour segment is then defined by

$D_{\alpha}=\sum_{i=1}^{n-1} w_{i-1} \cdot d_{i}$

To calculate the distortion measure for a pixel to be flipped, the contour segment passing through this pixel is extracted by a contour tracing method [7], after connected component labeling of the whole image. It is the 'original contour segment'. Similarly after flipping the pixel, the 'modified contour segment' is also extracted. From these two contour segments, the distortion measure is computed by taking the absolute difference of their curvatureweighted distances. Let $D_{\alpha}{ }^{\text {original }}$ and $D_{\alpha}{ }^{\text {modified }}$ be the curvature-weighted distances of the original and modified contour segments respectively. The curvature-weighted distance difference ( $C W D D)$ measure for the pixel is then given by:

$C W D D=\left|D_{\alpha}^{\text {original }}-D_{\alpha}^{\text {modified }}\right|$

\section{SUBJECTIVE EXPERIMENTS}

The purpose of the subjective test is to validate the high correlation between the model and the subjective ratings for different values of $C W D D$ measure. Using the Adobe Photoshop software, four characters 'A', 'B', 'E', 'S' are converted to binary images of size $128 \times 128$ pixels. These binary images, as shown in Fig. 1 are used as the original image set from which all test images are produced for the subjective experiments. It is difficult to produce a test image for each value of the $C W D D$ measure due to an insufficient number of flipping pixels of one particular value. To overcome this difficulty, we use 
the technique called binning to produce the test images. We divide the $C W D D$ range from $[0,8]$ into 9 bins. With the exception of the first one, each bin is of unity length in terms of $C W D D$ measure. The first bin consists of the pixels with $C W D D$ value equal to 0 . After careful experiments, for each bin we choose to flip a maximum of 10 and a minimum of 4 pixels in the original image to produce one test image. The distortion in the test image is the mean of the CWDD values of its flipped pixels and it is denoted by $C W D D_{\text {mean }}$.

From the original image set, a total of 34 test images are produced. The subjective assessment was performed by 25 observers. Each observer was asked to rate the amount of impairment in the test image with reference to the original image, on a discrete five-level scale ranging from 'imperceptible' to 'very annoying' as shown in Fig. 2. For each test image, the mean opinion score (MOS) is computed by taking the mean of the twenty-five subjective scores.

\section{RESULTS}

Here we show high correlation between the perceptual model described in Section 2 with the subjective test values obtained in Section 3. The subjective mean opinion score (MOS) was computed for each test image from the data obtained in the subjective experiments. In the plot of subjective MOS versus $C W D D_{\text {mean }}$ shown in Fig. 3, 26 points out of 34 are within the $95 \%$ confidence interval. The performance attributes Spearman rank-order correlation coefficients and correlation coefficients [8] are computed between subjective MOS and $C W D D_{\text {mean }}$ for all the test cases.

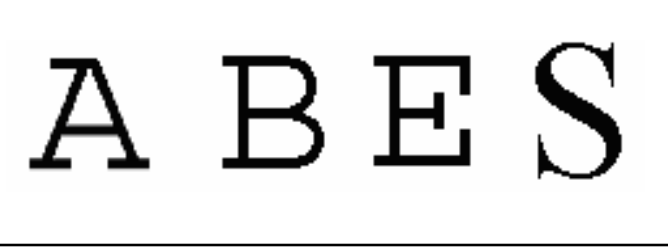

Figure 1: The set of binary images used for subjective experiments.

\section{Impairment}

Score

$\begin{array}{ll}\text { Imperceptible } & 0 \\ \text { Perceptible } & 1 \\ \text { But not annoying } & \\ \text { Slightly Annoying } & 2 \\ \text { Annoying } & 3 \\ \text { Very annoying } & 4\end{array}$

Figure 2: Rating scale

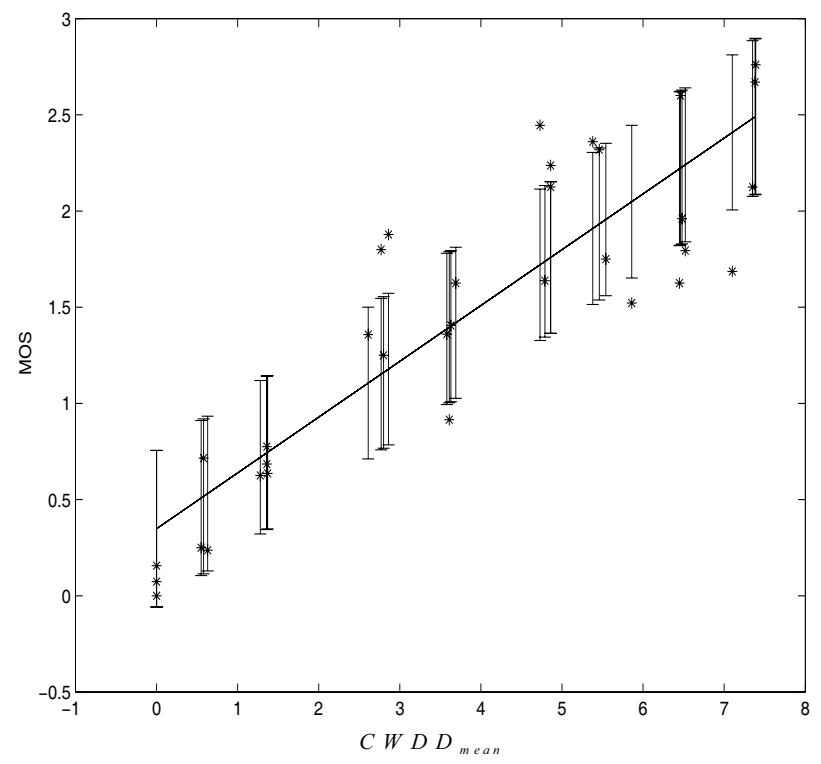

Figure 3: Subjective MOS vs. $C W D D_{\text {mean }}$ for all characters. Error bars indicate the $95 \%$ confidence intervals.

\begin{tabular}{|l|l|l|}
\hline Character & $\begin{array}{l}\text { Spearman ratik order } \\
\text { correlation coefficient }\end{array}$ & $\begin{array}{l}\text { Correlation } \\
\text { coefficient }\end{array}$ \\
\hline$A$ & 0.97 & 0.96 \\
\hline B & 0.88 & 0.94 \\
\hline E & 0.60 & 0.72 \\
\hline S & 0.95 & 0.93 \\
\hline All & 0.84 & 0.88 \\
\hline
\end{tabular}

Table 1: Performance attributes for all test cases. 
The recent development of various methods of modulation such as PCM and PPM which exchange bandwidth for signal-to-noise ratio has intensified the interest in a general theory of communication. A basis for such a theory is contained in the important papers of Nyquist and Hartley on this subject. In the present paper we will extend the theory to include a number of new factors, in particular the effect of noise in the channel, and the savings possible due to the statistical structure of the original message and due to the nature of the final destination of the information.

The recent development of various methods of modulation such as PCM and PPM which exchange bandwidth for signal-to-noise ratio has intensified the interest in a general theory of communication. A basis for such a theory is contained in the important papers of Nyquist and Hartley on this subject. In the present paper we will extend the theory to include a number of new factors, in particular the effect of noise in the channel, and the savings possible due to the statistical structure of the original message and due to the nature of the final destination of the information.

Figure 4: Top image is the original image of size $302 \times 410$ pixels. Bottom image with 1031 flipped pixels in CWDD range $[0,1]$.

The results summarized in Table 1 show that our perceptual model is highly correlated with the human perception in estimating distortion based on the flipping of a pixel. For all cases except in ' $\mathrm{E}$ ', $r_{s}$ and $\rho$ are above 0.8 . In case of 'A', 'B' and ' $\mathrm{S}$ ', the values are near or above 0.9 . So the model prediction is highly correlated to the subjective ratings. In Fig. 4, using the $C W D D$ model 1031 pixels are flipped in a text document image. The quality of the marked image is perceptually similar to the original one.

\section{CONCLUSION}

In this paper, we introduced a new perceptual model based on the $C W D D$ measure between two contour segments. Subjective experiments were performed with 25 observers with four characters 'A', 'B', 'E' and ' $\mathrm{S}$ '. Most of the model predictions (78\%) were within the $95 \%$ confidence interval of the subjective ratings. For the data set comprising of all the individual data sets, correlation coefficient was 0.88 and spearman rank order correlation coefficient was 0.84 . The experimental results validate the proposed model to be highly correlated to human perception, which would be useful for watermarking applications in binary images. Furthermore the proposed model has found to be particularly useful for sharplycontrasted binary images such as text, drawing and signature images.

\section{REFERENCES}

[1] S. H. Low, N. F. Maxemchuk, and A. M. Lapone, "Document identification for copyright protection using centroid detection," IEEE Trans. on Communication, vol. 46, no. 3, March 1998, pp. 372383 .

[2] S. H. Low, and N. F. Maxemchuk, "Performance comparison of two text marking methods," IEEE Journal on Selected Areas in Communications, vol. 16, no. 4, May 1998.

[3] E. Koch, J. Zhao, "Embedding robust labels into images for copyright protection," Proc. International Congress on Intellectual Property Rights for Specialized Information, Knowledge \& New Technologies, Vienna, Aug. 1995.

[4] D. Huang and H. Yan, "Interword Distance Changes Represented by Sine Waves for Watermarking Text Images," IEEE Transactions on Circuits and Systems for Video Technology, vol. 11, no. 12, December 2001.

[5] M. Wu, E. Tang, and B. Liu, "Data hiding in digital binary images," Proc. IEEE Int'l Conf. on Multimedia and Expo, Jul 31-Aug 2, 2000, New York.

[6] Haiping Lu, Jian Wang, Alex C. Kot, and Yun Q. Shi, " An objective distortion measure for binary document images based on human visual perception, " Proc. Int. Conf. Pattern Recognition, vol. 4, Quebec, Canada, Aug. 2002, pp. 239--242.

[7] T. Pavlidis, Algorithms for Graphics and Image Processing, Computer Science Press, Rockville, Maryland, 1982.

[8] D. Anderson, D. J. Sweeney, T. A. Williams, Introduction to Statistics: An Applications Approach, West Publishing Company, 1981. 